\title{
Perceptions of clinical leaders and managers of inpatients with obesity in an Australian public health service
}

\author{
Alison Qvist ${ }^{1}$, Fiona Pazsa ${ }^{1}$, Danielle Hitch ${ }^{1,2}$ \\ ${ }^{1}$ Allied Health, Western Health, Sunshine, Australia; ${ }^{2}$ Occupational Therapy, Deakin University, Geelong, Australia \\ Contributions: (I) Conception and design: All Authors; (II) Administrative support: A Qvist, D Hitch; (III) Provision of study materials or patients: \\ All Authors; (IV) Collection and assembly of data: A Qvist, D Hitch; (V) Data analysis and interpretation: All Authors; (VI) Manuscript writing: All \\ Authors; (VII) Final Approval of manuscript: All Authors. \\ Correspondence to: Alison Qvist. Western Health, Footscray Hospital, Gordon Street, Footscray, Victoria 3011, Australia. Email: Alison.Qvist@wh.org.au.
}

Background: In 2014-15, 63.4\% of Australian adults were overweight or obese, with 25\% categorized as obese. Internationally, people with obesity are reported to experience inadequate quality of care, increased length of stay, more adverse events and higher costs of care. There are unique challenges associated with ensuring this cohort is provided with safe care which promotes their personal dignity. The aim of this study was to describe the experience of clinical leaders and managers of care provision to people with obesity, during inpatient admissions to an Australian public health service.

Methods: A purposive, convenience sampling method was utilised, resulting in the recruitment of 17 participants. Data was collected via semi-structured interviews in the workplace, which were all digitally recorded for verbatim transcription. All data was subjected to thematic analysis, with identified codes reorganised into overall themes.

Results: Five overarching themes were identified, four of which are discussed in this publication. The main themes and subthemes were (I) resource allocation (incorporating inequality, economic resources, human resources and physical resources); (II) service context (incorporating understanding 'bariatric', physical/ built environment, staff knowledge and skills, and organisational culture; (III) care transitions (incorporating transitions in care, communication and organisational processes; and recommendations for best care for people with obesity.

Conclusions: The experience and perceptions of participants reflect the issues they prioritised in their respective roles, and confirm that providing care for people with obesity has significant policy and practice implications. These issues cannot be considered in isolation, with significant overlap and interdependence was evident. Participants also described the positive outcomes and progress which could be achieved when organisations take direct action to improve the care they provide to people with obesity.

Keywords: Obesity; bariatric; service provision; perceptions; clinical leadership

Received: 16 July 2020; Accepted: 22 January 2021; Published: 25 June 2021.

doi: 10.21037/jhmhp-20-98

View this article at: http://dx.doi.org/10.21037/jhmhp-20-98

\section{Introduction}

Obesity and overweight refers to abnormal or excessive fat accumulation, that poses a risk to health (1). Both states are global and growing public health issues, with international research indicating their impact will continue to rise with increasing community prevalence (2). Obesity is also a leading cause of mortality from cardiovascular disease, diabetes, kidney disease (3), and contributes to $12.5 \%$ of hospital admissions in Australia (4). However, there have been few studies of healthcare worker experiences of providing care to people with obesity. 


\section{Identifying obesity}

The World Health Organisation defines obesity as having a body mass index (BMI) $\geq 30 \mathrm{~kg} / \mathrm{m}^{2}$, with morbid (or class 3) obesity defined as a BMI $\geq 40 \mathrm{~kg} / \mathrm{m}^{2}$ (5). The term 'bariatric' refers to medical intervention aimed at weight loss (6), however it is widely used in clinical practice to refer to patients with obesity. As per recommendations around person first language (7), this paper uses the term people with obesity unless 'bariatric' appears in direct participant quotes.

Whilst a high waist circumference $(>102 \mathrm{~cm}$ men, $>88 \mathrm{~cm}$ women) is associated with increased obesity related morbidity, there is currently no evidence based, standardised or even consensus approach to waist measurement circumference (8). Cut off weights may be used to guide maximum loading and lifting (9), however bodily proportions are possibly more relevant in clinical practice. Variable skills in calculating BMI are another recognised barrier to the identification of people with obesity, with a study of 834 Nursing and Medical staff finding the majority did not know how to calculate BMI or diagnose obesity (10). The potential impact of underidentification was highlighted in a retrospective study of 540 inpatient admissions, which identified a discrepancy between the proportion of patients with $\mathrm{BMI} \geq 30$ (31.4\%), and specific documentation of obesity at admission (23.5\%) or discharge (12\%) (11).

\section{Clinical issues and models of care when caring for patients with obesity}

Previously identified clinical issues when caring for people with obesity include equipment and the built environment, skin and wound care, manual handling and mobilisation, and nutrition. The availability of adequate equipment and an enabling built environment is essential when caring for people with obesity. Australasian health facility guidelines (12) specifically recognise the need for adequate space for manoeuvring bariatric equipment and furnishing, and structural reinforcement for specialist lifting equipment such as hoists. Examples of specialised equipment include transfer devices, pressure relief mattresses, moisture management sheets, longer needles, larger blood pressure cuffs and plus size hospital gowns (13). There may also be challenges associate with the design of equipment for people with obesity, which are often simply scaled up rather than adapted to bodily proportions (14). As highlighted by Nowicki et al. (9), hospitals are generally ill equipped to manage people with obesity, and given the purchase costs may be up to triple that of standard equipment, these resources are perceived as highly specialised.

Patients with obesity experience altered skin structures including greater water loss, dry skin, increased erythema and lower microvascular reactivity $(15,16)$. They also have deeper skin folds, that may promote bacterial and fungal growth and increase the risk of skin tears, pressure and friction injuries (15). Toileting poses particular challenges for skin care, and these issues may be exacerbated by poor staff skills and a lack of time for skin care activities $(15,17,18)$. Skin care can also be promoted through the use of appropriately sized equipment, and regular mobility particularly targeting areas of fat deposition (19). No validated tools current exist for skin assessment with these patients, and instigating pressure injury prevention strategies for all people with obesity has been recommended $(15,20)$.

Specific skills are required to safely manually handle patients with obesity, with previous research and Australian guidelines $(21,22)$ highlighting the need for training in risk assessment and risk management, power equipment and patient centred approaches which enable dignity and independence. Both healthcare workers and patients have reported accidents and incidents arising from poor or ineffective practices, which have in some cases resulted in injury (22). A mixed methods study (23) from the United Kingdom reported the majority of healthcare workers struggled to access specialised equipment and training, and worked in services without bariatric manual handling policies. Without organisational policies and procedures, the simple availability of equipment and enabling built environments is not sufficient to support effective manual handling practices for patients with obesity.

While nutrition is a core aspect of obesity, restricting intake for these patients may exacerbate the impact of malnutrition arising from a poor diet pre-admission (15). People with obesity may lack vital nutrients required for healing (15), further increasing their risk of skin breakdown and pressure injuries (19). Intensive care research also found that people with obesity experience greater metabolic rates, protein catabolism, and muscle mass deterioration (24), which was associated with a higher risk of deconditioning and risk of mortality. Green et al. (25) recommended a nutritionally adequate diet be provided during admission, with further follow up provided if the patient expresses motivation for weight loss after recovery (25). Early 
Dietitian intervention and nutrition support has also been recommended, using specific obesity adjusted requirement calculations (24).

Models of care have been developed to provide a comprehensive and coordinated approach to caring for people with obesity. In some locations, multidisciplinary teams including nurses, allied health clinicians, equipment and occupational health and safety $(\mathrm{OH} \& \mathrm{~S})$ experts, have formed 'bariatric' teams or working groups $(9,26,27)$. These groups provide comprehensive assessment; procure and manage equipment and environmental issues; and promote continuity for patients throughout admission (28). Specialist 'Bariatric' nurse positions have also been proposed, along with the routine recording of patient weight in all incident reporting, mapping patient journeys and the instituting of mandatory workforce education around the care of people with obesity (18). There have been very few evaluations of these new models of care to date, with key performance indicators and outcomes for patients with obesity yet to be established (28). However, a case study (29) has reported a 68-day reduction in admission length for a patient with obesity following the introduction of a Bariatric Care Team between consecutive admissions.

\section{Rationale}

While a small amount of research has explored clinical issues when caring for people with obesity, the healthcare worker experience of this care is largely unexplored beyond studies of general perceptions linked to the phenomenon of 'weight bias'. This is particularly true for clinical leaders and managers, who may exert a strong influence on direct care delivery via their broader perspective on organisational culture and practices. The aim of this study was to describe the experience of clinical leaders and managers of care provision to people with obesity, during inpatient admissions to an Australian public health service. We present the following article in accordance with the MDAR and COREQ reporting checklist (available at http://dx.doi. org/10.21037/jhmhp-20-98).

\section{Methods}

\section{Study context}

This study was conducted in an Australian tertiary public health service, located in a diverse and rapidly developing community, with a higher than average prevalence of mental health issues, diabetes and obesity $(30,31)$ The organisation encompasses three acute hospital campuses, along with outpatient and community services (30). The organisation had already identified the need to improve the quality of care provided to patients with obesity, establishing an executive sponsored 'Bariatric' working party of clinicians and managers from diverse clinical areas. The working party was focused on driving improvement in regards to; (I) coordination, communication and flow; (II) patient experience; (III) equipment; (IV) education, training and research; and (V) data, demographics, infrastructure and planning.

A referral based multidisciplinary consultation service, known as the Bariatric Assessment Team (BAT) has also been established. This team works with patients who are morbidly obese, whose weight exceeds the safe weight capacities of standard hospital equipment or are experiencing functional problems related to their obesity. Following comprehensive assessment, the BAT provide their treating team with recommendations and consultation around equipment, bed allocation, manual handling, skin integrity, referrals and discharge planning.

\section{Researcher stance}

A previous methodology review of studies on people with obesity (32), found reflexivity and researcher stance was often neglected. All researchers completed the Attitudes Towards Obese Persons and Beliefs About Obese Persons Scales (33), which are both valid and reliable measures of weight bias (34). Their response to these scales helped to identify individual assumptions, forming the basis of iterative peer discussions throughout the course of this study.

The first author (AQ) is a Dietitian and Clinical Informatics Analyst. She initiated this study during her Master's in Health Services Management, and also subsequently undertook a project role to improve care for patients with obesity at the organisation. AQ was relatively naïve to the negative attitudes and beliefs towards people with obesity prior to this study. She believes that health comes in different sizes, and the biggest impact on health outcomes is a person's health related behaviours rather than weight.

The second author (DH) is an occupational therapist and academic, with significant experience with qualitative research. She was the allied health research and translation lead at the organisation, and also held an academic post at a 
nearby university. DH held predominantly positive attitudes and beliefs about people with obesity, which she partly attributes to her own lived experience with this condition. However, her past clinical experience also provided some empathy with clinicians, who she believed are usually doing their best with the resources they are provided.

The third author (FP) is a Physiotherapist with nearly 20 years clinical experience. Through frequent interactions with patients with obesity, she became interested in improving the care provided to these patients, and was a founding member of the organisation's Bariatric Working Party. She is currently completing a higher degree by research on this topic, with the longer term aim of developing an improved model of care for people with obesity during inpatient admissions. FP lives a healthy lifestyle and encourages others to do the same, however acknowledges the challenges that can lead to obesity

\section{Methodology}

The Model of Information Power (35) provided a framework for methodological design in this study. Information power indicates the more information the sample holds specific to the study aims, the fewer participants needed for understanding of the phenomenon inclusive of variance. Samples must include people with experiences, or properties specifically related to the study aim (35) with those including previously unexplored experiences considered especially powerful. Semistructured interviews prompts based on existing evidence, and the advanced interviewing techniques of the researches supported the collection of quality interview dialogue (35). Rigour was also enhance by addressing of methodological issues raised about previous studies with people with obesity (32).

\section{Recruitment and data collection}

A purposive, convenience sampling method was utilised, to recruit clinical leaders and managers working in services that care for patients with obesity at the organisation. Potential participants identified included members of the 'bariatric' working party, clinicians in ward-based leadership roles and other key stakeholders identified by the researchers. Participants were invited via email by a researcher with whom they had no prior professional relationship and were provided a written plain language statement. Participants were informed that the intention of the study was to utilize the findings to drive service improvement and ensure best care for people with obesity seen at the organisation.

All semi-structured interviews were undertaken by DH or AQ between February and May 2019, and lasted between 25 and 65 minutes. Interviews occurred in private meeting rooms within the organisation and only study participants and researchers were present. Interview prompts were piloted with non-participant colleagues, with minor modifications to wording made on the basis of feedback. Interviews were digitally recorded for verbatim transcription, which occurred within 2 to 4 weeks. No field notes were taken. No repeat interviews occurred. All data was de-identified at transcription, with participants given an alphabetical pseudonym for privacy (i.e., Participant A). Completed transcripts were provided to all participants for member checking, with only one returned with grammatical and spelling corrections.

Thirty-three clinicians were invited, of whom $52 \%$ $(\mathrm{n}=17)$ consented and were interviewed. The majority were allied health professionals $(n=9)$, or nurses $(n=7)$, with a single occupational health and safety practitioner. No participants dropped out.

\section{Data analysis}

Statistical analysis: due to the qualitative research methodology, there was no statistical analysis on this dataset.

Thematic analysis was undertaken to understand the participants perspectives, beginning with line by line coding on the Dedoose platform (36). One researcher (DH or AQ) analysed alternating transcripts, producing codes for their interpreted meaning for the text. The non-coding researcher then reviewed each transcript for understanding and consistency of code application. Very few disagreements were found, and were resolved via consensus discussion. All researchers then met for the next level of thematic analysis. All codes were printed out, discussed and rearranged to identify overarching themes, four of which are reported here. The fifth theme, regarding attitudes towards patients with obesity, is reported in a separate publication. The results of the study were presented back to the Bariatric Working Party and feedback sought. The Consolidated Criteria for Reporting Qualitative Research (COREQ) were utilised to maximise the quality of reporting for this study (37).

Rigour in qualitative research is indicated by its adherence to trustworthiness criteria (38), and several verification methods were included in the study design. 


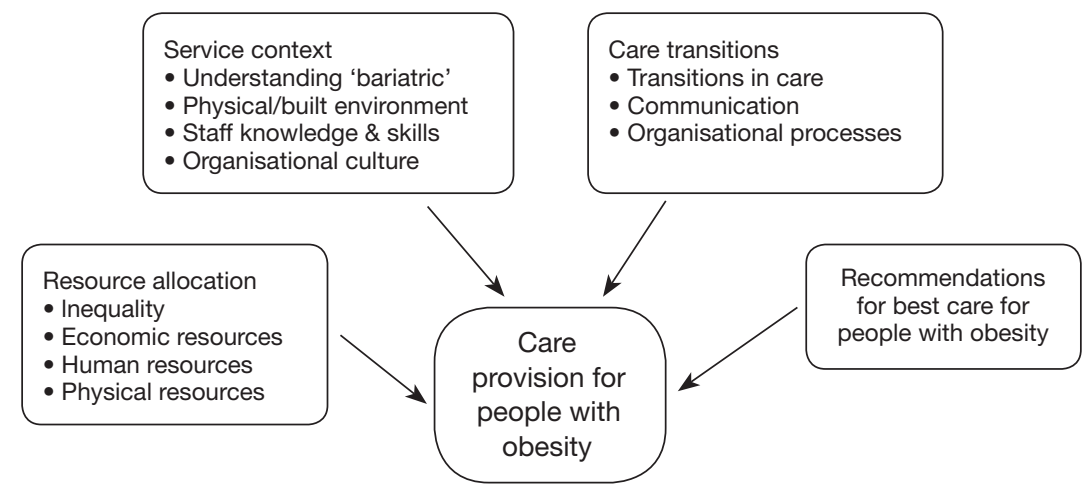

Figure 1 Identified themes and sub-themes.

Along with member checking and reflexivity, the review of coding for consistency and consensus enhanced credibility. Access to regular peer review within the research team was complemented by peer review from other members of the Allied Health Research Leadership group at the organisation. DH is an experienced researcher, and provided supervision and guidance to AQ and FP. All study documentation has been stored securely, and is available for audit.

\section{Ethical statement}

The research was conducted in accordance with the Declaration of Helsinki (as revised in 2013) and the Harmonized Tripartite Guideline for Good Clinical Practice from the International Conference on Harmonization. This study was reviewed and approved by the participating healthcare originations office of research (HREC/18/ WH/47094). All patients enrolled completed the informed consent form.

\section{Results}

As shown in Figure 1, the identified themes were: resource allocation; service context; care transitions; and recommendations for best care for people with obesity.

\section{Resource allocation}

Equality was a very important aspect of resource allocation for participants. On one hand, they believed care should be provided equitably to everyone, and people with obesity should be treated no differently. Specific models of care may not be warranted if all patients are treated as individuals and receive person-centred and personalised care. However, participants also acknowledged that patient cohort are more complex and often require greater resources. Many recounted incidents when patients with obesity received lower quality care compared to their other patients, attributing these issues to limited access to assistive equipment, inappropriate physical environments and inadequate staffing. These barriers were perceived by some to cause patients with obesity to receive less intense therapy, potentially delaying their recovery. Their complex needs were also perceived as a challenge, as people with obesity do not fit neatly into existing service structures, requiring advanced and creative clinical reasoning from healthcare workers. As a result, clinicians often required the assistance and support of additional colleagues to deliver care, which was not always available when required.

Participants also reflected on the increased treatment costs for patients with obesity, in terms of clinician time, equipment and infrastructure. A lack of funding was believed to contribute to these patients missing out on basic resources, such as appropriately sized hospital gowns, safe and secure seating and accessible toilets. Participants believed the ongoing lack of access to these resources has a negative impact on the patient's physical and mental wellbeing, contributing to further functional decline, suboptimal outcomes and delays in discharge. While bariatric equipment is much more expensive than standard items, clinicians overwhelmingly perceive that investing in their procurement results in significant downstream savings, for both the patients and the organisation. However, tensions were also identified for participants in balancing quality care with financial sustainability.

Time and staffing were very prevalent concepts, intertwined with every aspect of care for people with 
Table 1 Sub-theme summary for resource allocation

Inequality

- "It's the same model as every other person, it may just look a bit different. You can't have one size fits all. If you don't know the person in the bed then you really don't know what the best care is for them" (C)

- "They wouldn't necessarily get the same access to our group therapy or hour hydrotherapy because it takes so long to set them up at, the length of their sessions would probably need to be shorter" (B)

- "The amount of therapy she could actually tolerate from a fatigue point of view was really quite minimal ... it's not just your average patient that you can use pattern recognition and remember how you managed it last time" (B)

Economic resources

- "I know we've been ... advocating for more equipment to be available, that's purchased through the hospital for years now and it was just quite "too expensive" even through in my mind it would have paid for itself a million times now" (C)

- "Well this is going to kick the budget but that's irrelevant, it's about safety and the patient comes first" (E)

Human resources

- "It takes time to actually lift the apron up even when they are in the bed to give it a really good clean, ... it's actually going to take a couple of people helping you to do it properly" $(\mathrm{H})$

- 'We've had patients waiting on one of the units waiting to get to rehab because we're at capacity of how many patients we can take, whether that's hoist dependent or bariatric in the mix" (E)

- "Obviously we have to make sure we've got the resources as in staff, so you're not gonna put staff at risk" (O)

- "You might be calling 7-10 companies to find something you need instead of 1-2 so the time required to look after this group and do our jobs properly takes a lot longer than other populations" (C)

Physical resources

- "Having access to the right equipment is a big issue" (M)

- "The beds are difficult to find sometimes ... we're cruising around all day looking to retrieve stock, so often we see where those things are sitting in back corridors or empty rooms" $(\mathrm{J})$

- 'He said 'yeah there's not chairs and if I sit l'll get stuck in them'. So this guy's regularly coming to the outpatient clinics but doesn't have anywhere to sit, could be waiting for up to 2-3 hours and stands the whole time" (K)

- "You would have the client on the bed and the physio couldn't get on as well and a lot of work we could do to try to get their muscles stronger is us sitting on the bed or kneeling on the bed with them" (B)

- "I mean nobody looks decent in the gowns, but you want to be at least covered" (O)

obesity. The body habitus and mobility of these patients' increases the time required for self-care tasks, and the number staff required to perform these tasks safely. Clinicians highlighted potential risks to both patients and staff in circumstances of under-resourcing, and the importance of prioritising safety.

The most consistently identified resource barrier was the lack of availability of suitable equipment. Available bariatric equipment in the organisation is shared between services, and can be difficult to locate when required. Even when available, the dimensions and structural features of bariatric and medical equipment may themselves be a barrier to quality care. Participants expressed particular annoyance at the lack of suitable furniture, clothing and footwear, which many felt was a key barrier to treating people with obesity with dignity during their hospital admission or when attending for outpatient care.

Table 1 provides an overview of direct participant responses regarding resource allocation, conveying the overall perception of widespread scarcity having a negative impact on best care for people with obesity.

\section{Service context}

There was noticeable variability in perceptions around the identification of people with obesity at the organisation. 
Many discussed BMI as a key variable, some identified weight cut-offs, while others spoke of girth. However, most participants emphasised that any of these concepts wasn't sufficient on their own to meaningfully define obesity. Some clinicians didn't address anthropometrics at all, focusing only on the patient's functional and occupational capacity. For these participants, obesity was only a clinical problem if it prevented performance of valued activities for the patient. This suggests there continues to be important challenges for the consistent identification of patients with obesity requiring specialised care during inpatient admissions.

The built environment in hospitals was generally perceived as inaccessible and exclusionary for people with obesity. This barrier was perceived to be evidence from the moment of arrival at hospital, and was believed to have major negative impacts on both admissions and discharges. Room dimensions were often highlighted by participants (as bariatric equipment generally requires more space), which is exacerbated at this organisation by ageing hospital infrastructure on some sites. This issue was particularly noticeable when patients were transferred from a unit with better facilities to an older part of the hospital, which in some cases was believed to have precipitated clinical deterioration. The ongoing use of bariatric equipment post discharge was also recognised as problematic, as many people with obesity are unable to modify their accommodation to meet their needs.

Overwhelmingly, participants felt it was every staff member's responsibility to enable best care for people with obesity. Clinicians at all levels (including leadership and management) were perceived as playing an important role, as these patients may access services from any area of the organisation. Specialist support to treating teams, from continence specialists, respiratory physicians, wound care nurses, clinical psychology and occupational health and safety representatives, was particularly valued by clinicians as a key aspect of providing best care. However, many participants also perceived an overall lack of role clarity, with differing priorities around optimal care and definitions of good outcomes reported between disciplines. This could be managed within effective multidisciplinary teams by keeping a broad perspective of the patients social and functional context during decision making, however the medical model is still perceived to dominate inpatient services.

The need to develop knowledge and skills about best care for people with obesity for staff outside the Bariatric Working Party was also widely discussed. Many participants reflected that with the increase of obesity prevalence globally, 'it is going to be bread and butter for us' (E). However, current workforce knowledge and skills was observed to be suboptimal, with most healthcare workers thought to be underprepared, leading to feelings of anxiety and stress which could ultimately contribute to withdrawal from working with this cohort. Opportunities for increasing capacity were identified across a range of topics, including using appropriate language when discussing weight, sourcing bariatric equipment and consumables, and effective manual handling and risk reduction techniques. Widely and regularly available training in specific clinical skills was also perceived to be a priority, including skin care practices, pressure risk reduction and venous access when caring for people with obesity.

The initiatives already taken at the organisation (such as the Bariatric Working Party and BAT) were also believed to have contributed to shifting the organisational mind set and increasing awareness. Attitudes and motivation within the organisation was reported to be becoming increasingly positive by many participants, who were hopeful of sustained improvements. There were also frequent references to the need to facilitate best practice on a consistent basis for meaningful change to be sustained. Leadership at the senior and executive levels of the organisation was identified by most participants as supporting the maintenance of patient centred approaches in the face of organisation pressures. Governance structures were also recognised as facilitators, from local working parties up through to committees aligned to the organisations best care framework. These frameworks promoted links to be made between the needs of people with obesity and other vulnerable groups, reporting and dissemination of visible changes in practice and a more systemic approach to the initiatives being introduced. However, participants also acknowledge these were still developing, and needed wider roll out to be evaluated and their effectiveness fully understood. Development was therefore perceived to be emergent, and with widespread confirmation from the participants that change to organisational culture is extremely challenging.

Table 2 displays verbatim participant quotes related to the service context, which indicates that while the identification of these patients remains a challenge, there was considerable commitment to and hope around ongoing improvements to care for patients with obesity at the organisation.

\section{Care transitions}

Many participants reflected on the regular delays that 
Table 2 Sub-theme summary for service context

Understanding 'bariatric'

- "You could have some, two people of the same weight, someone who is tall and can use standard equipment and someone who's shorter and sort of wider" (C)

- 'They're 220 and 230 and you'll go down to see them and they're literally walking past you after having a shower and you say to the nurse 'what, why did you want us to come help, is there an issue?' 'Oh no, we just looked at the weight'” (D)

Physical/built environment

- "There was a heap of stuff he had to work through with me and our manager to get him parking, a place where he felt comfortable so access to the building before you even get care" (B)

- 'When you've had really good access to an appropriate bathroom and then you go what is supposed to be the next, the best step on your journey ... and you're told no sorry you'll have to use a pan, that's not really appropriate" (G)

- "He cannot get home unless he's on some crutches or a stick, as a frame will not fit through any of the doors ... but he's in a rental, you know those, you can't made any mods to the rentals" (E)

Staff knowledge and skills

- "I think needs to start with our leaders and like our sort of directors of nursing, our ops managers, our managers of all the different allied health teams, I think everybody plays a role right up to the Personal Support Assistants and their managers, the directors and things like that because it effects every team member that works at [organisation]" $(\mathrm{N})$

- "At the moment we don't do any specific staff education around bariatric care. I think we're learning as we go what might need to be included from that perspective" (M)

- "When we first started 6-8 months ago, we had to do a lot more facilitation around providing best care for this patient group. As we've gone along we've educated the staff on the ground and now we find that before we even get to see the patient a lot of the recommendations that we would have put in place are already in place" (M)

Organisational culture

- "There's so many people at [organisation] that are really passionate about this, which I think drives it so well" (I)

- "I think we need to work on is the consistency, and having that really across the board, every day, for every patient, from everyone and that's the challenge" (L)

- "I think that (the organisation) has acknowledged the issue and is really keen to be a leader in the area, so hopefully we can come up with some more concrete answers or models of care and be able to share them with other organisations" (M)

- "I just really hope that something gets done you know sooner rather than later ... I've sort of hoped that something would be done before this but I know these things take time" (F)

occur in transitions within the service for patients with obesity. The planning involved in obtaining bariatric equipment and arranging a suitable treatment environment contributed to these delays and often impacted negatively on continuity of care. Participants described these patients as being completely unable to access the optimal service for their care in the organisation, or experiencing timely transfer but then poor outcomes as the receiving service was inadequately equipped for their needs. Participants described experiencing significant frustration at these transition issues, believing they often resulted in people with obesity missing opportunities for timely discharge.

Transitions of care included pre-admission locations and post-discharge destinations in the community. Participants believed patients with obesity are frequently delayed or did not access available and potentially preventative community services prior to admission, and acknowledged there were many reasons outside the patient's control which contributed to this problem. Planned admissions were suggested as an opportunity for better preparation, as were closer partnerships with external providers and processes (such as receiving forewarning from the ambulance service when a patient with obesity is coming to the emergency department). However, most participants were more concerned about discharge, due to frequently encountered problems with this transition due to inadequate resources (such as transport and accommodation). Companies providing bariatric equipment during admissions were also 
Table 3 Sub-theme summary for care transitions

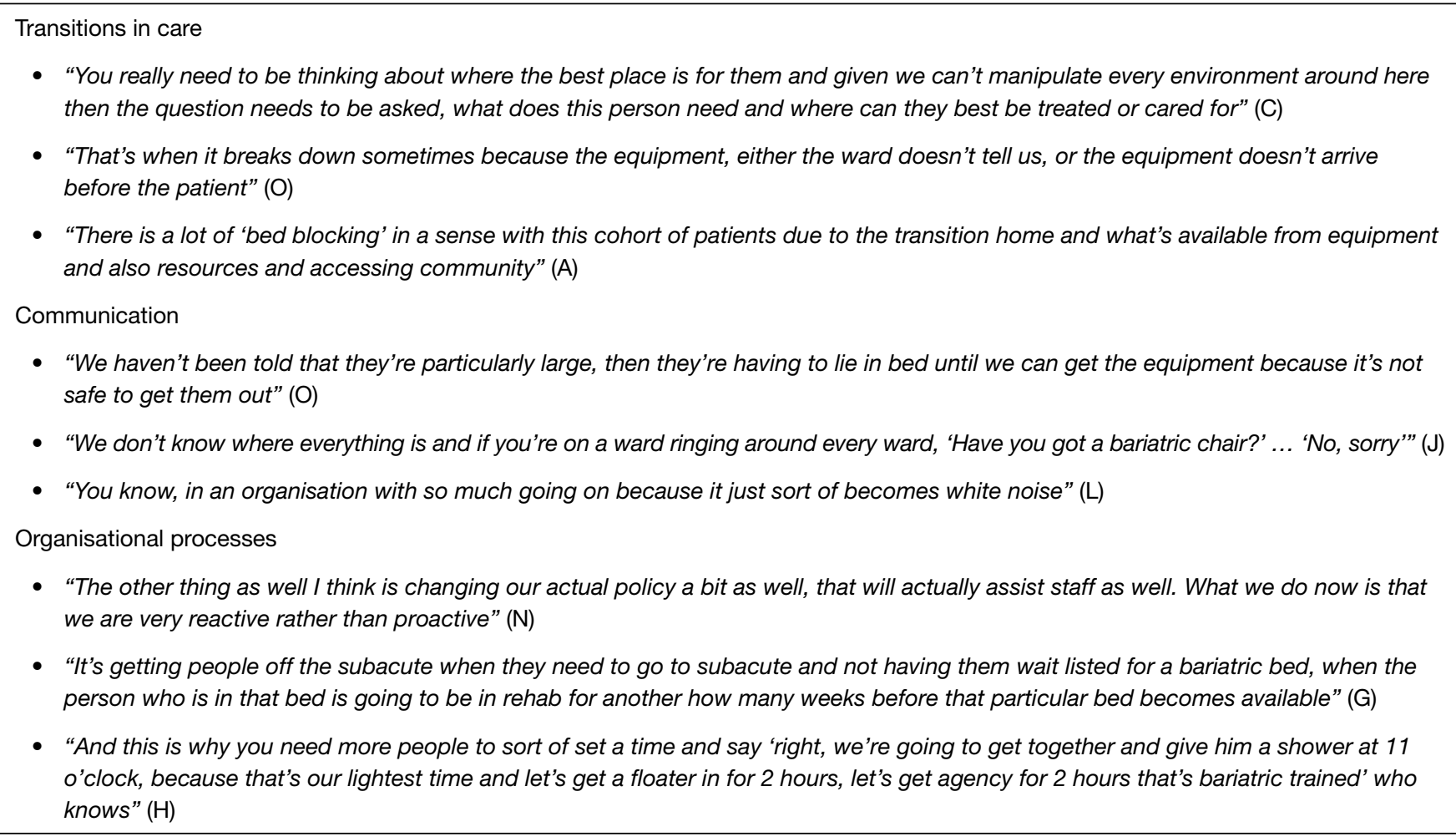

reported to refuse to supply it in the community, due to perceived occupational health and safety risks.

Communication was another key factor in care transitions identified and discussed by participants. Poor inter-service communication, both within and external to the organisation, frequently obstructed continuity of care. Many participants' recounted incidents where services had to recommence sourcing required equipment, consumables and referrals, despite their need being well established in the previous service. Participants linked this to the lack of overall resource coordination systems in public health services, and/or healthcare workers not understanding how to use the existing systems. Many participants perceived the formulation of clear and accessible organisational processes and procedures as an important communication strategy, particularly in regards to identifying these patients, appropriate inpatient environments, and relationship building.

As summarised in Table 3, the participants generally perceived care transitions to be a crucial, but poorly executed aspect of care for patients with obesity. Organisational processes and communication were both identified as key factors which could exert both positive and negative influences on continuity of care.

\section{Recommendations for best care of people with obesity}

This was a distinct and coherent theme, focused on participant's perceptions around how to ensure continuous improvement. The recommendations broadly aligned with challenges and issues identified in the previous themes, consolidating and extending on these findings. The actions suggested by participants are shown below in Table 4 . Overall, participants wanted to see progress in the care delivered to these patients so that 'when the patients present, we just know what we need to do within the right governance framework' (K). However, some areas provoked greater ambivalence or discord in responses than others.

The issue of whether people with obesity should be treated as a distinctive patient group provoked responses that indicated two opposing perspectives. Some participants advocated for the development of a specialist service; 'like a purpose specialist clinic I think where as a multidisciplinary team treatment is actually offered within that clinic rather than just consultation' (B). However, others cautioned against the formation of 'bariatric' clinics, primarily due to perceptions 
Table 4 Recommendations for best care of people with obesity

Recommendations

On-going implementation of multidisciplinary approach to care for people with obesity

- "The wound care nurse will look at something else and see the smallest of smallest red marks ... then the physio will then come in and look at maybe a therapeutic process, the OT will then come in and see things probably I don't have that level of expertise around. That multidisciplinary approach ... it's unbelievable" (K)

Continuation of the Bariatric Assessment Team (BAT), with a progressive shift from organisational capacity building to specialist consultation for patients with complex care and coordination needs

- "You would hope in a sense that over time that may not necessarily be needed because basically you skill up staff that it's become such standard practice" (E)

Greater inclusion of patients with obesity in workforce development, care design and delivery

- 'I think it's using the patient story, it's about how do we change staffs perception in terms of positively getting them to go, what can you do? ... I think co-design is good, I think that's a really strong way to go down ... I think only these people have a complete understanding of what it's like" (E)

- "So asking what they think they need to be able to continue to look after themselves to the best of their ability and be cared for and comfortable on the ward" (C)

Early, consistent and effective identification of patients with obesity via the introduction of an electronic medical records alert

- "Having something on their file that will raise, maybe they are going to have these extra needs, maybe we'll have to be involved a bit quicker" (D)

Development and pursuit of longer-term organisational strategy, including investment in equipment procurement and distribution

- "Here would be a box on a shelf that would have gowns in it, have the hover mat, bariatric hover mat ... little bits and pieces that you could come and get a pack. Like if somebody dies, you get a mortuary kit off the shelf" (J)

- "Whether we have some initiatives as well about being quite flexible and dynamic at ward levels about how we interact and engage and just thinking strategically about how we do therapy or those sorts of things" (A)

Provision of resources to enable clinicians to make practice changes, including local practice leaders or champions and amended policies and procedures

- "You need somebody with the drive that's gonna keep this momentum going ... they seriously need to look at a dedicated clinician in that role" (J)

- "Really good policies and procedures around how we care for bariatric patients so things like risk assessment, when the patient arrives, manual handling risks, how we reposition patients, full head to toe risk assessment, so your pressure areas and wound care" (I)

- "More of a pathway that you can tick a box, something that's easy for people to work their way through because I think sometimes it's just too complex for them ... so they just go and give up because they just go it's too hard" $(\mathrm{H})$

Proactive community care for people with obesity to prevent hospital admissions, including partnership with general practitioners and developing specialised outpatient clinics

- "That would be another way of having a stepping stone between primary and tertiary health care which I think needs to be a very significant multidisciplinary clinic" (C)

- "So our next part of the project is linking GP's that treat these patients ... (however) a lot of these patients don't have access to doctors" (K)

of inequality; 'It's not like bariatric patients all sit in this pile and everyone else all sits over here because it's not about whether you're bariatric ... its whether or not you're getting the right care at the right time with the right equipment and you know moving through the system appropriately' (G).
Equipment was also a contentious area in regards to recommended changes to practice and access. Initiatives to improve collaboration between equipment suppliers and the organisation were previously undertaken, but without success. When reflecting on attempts at collaboration 
with equipment companies, Participants highlighted the tensions between the profitability goals of the companies, versus the cost minimisation imperative of the public health system. Purchasing a stock of bariatric equipment, owned by the organisation and ready for deployment, was a consistent suggestion, as was the provision of 'bariatric kits' to each service including essential non-equipment items. There was also an upcoming chance to influence the design of updated infrastructure at this organisation, which participants perceived as a unique opportunity to provide a more accessible and inclusive built environment for people with obesity. However, the provision of a better environment was also recognised to potentially require additional staffing resources; 'We need to look at nursing rates to go with that as well, because it doesn't matter how many beds you've got if you don't have the nursing staff to also make that bappen' (G).

\section{Discussion}

This study described the experience of clinical leaders and managers of care provision to people with obesity, during inpatient admissions to an Australian public health service. While the themes identified align with some existing research about care for people with obesity, some of these findings offer a novel insight into the experience of these clinicians.

Equipment and infrastructure issues were consistently raised, in regards to both resource allocation and the organisation context. While this study focused on clinical perspectives, research directly focused on the experiences of people with obesity has also identified a theme of 'struggling to fit in' to inaccessible health environments that do not provide appropriate equipment and space (including time) for providing best car (39). Clinicians in other research studies have also expressed the belief that patients with obesity cannot be treated safely, comprehensively and with dignity without adequate equipment and infrastructure $(9,24,40)$. A qualitative study by Lumley et al. of nonleadership or management clinicians (41) included their perceptions of the negative impact poor access to furniture and equipment has on timely treatment and complications. Significant auditing, planning and purchasing are required to ensure these resources are available to meet clinical demand, and the prevalence of this issue indicates that healthcare organisations worldwide are struggling to provide the equipment and infrastructure that people with obesity need. It also supports the impression that 'bariatric' equipment in particular is still perceived as specialised, rather than a requirement of regular practice.

The varied understandings of 'bariatric' and/or obesity reflect disciplinary differences to some degree, but have a significant impact on care delivery for these patients. Quantitative benchmarks and weight limits are relatively easy to apply, however are increasingly recognised as being arbitrary rather than evidence based (42). A critique by Stevens (43) also highlights that the impact of significant gender differences in body fat, ethnicity and activity levels are not considered in BMI calculations. However, she acknowledges that BMI scoring has the advantage of being a low cost and easy to complete clinical measure. Quantitative measures are therefore likely to remain a feature of clinical definitions of obesity, however functional and anthropomorphic perspectives also have much to offer.

Another finding from this study that reflects prior research was the emphasis on, and valuing of, a multidisciplinary approach to care for people with obesity. The focus on skin and wound care, and nutrition found in the literature review as not as prominent in the participants perceptions, but were mentioned in passing. Given the influence of contextual factors on implementation in healthcare (44), some variation in focus and priority is expected between healthcare organisations and systems, but does pose a challenge to the development of generalised models of care. Many examples of multidisciplinary approaches to the care of people with obesity are available in the literature (9,26-28), although few have been evaluated for their ability to enable patients to receive a personalized package of care that meets their specific constellation of needs.

The BAT team at this organisation was universally perceived positively, despite some varying opinions around its longer-term role. Governance structures that allow for escalation and a coordinated approach to clinical challenges must support such models of referral based multidisciplinary consultation services. The implementation of new models of care also requires significant cultural change and investment for the organization, necessitating support from clinical leadership, management and executive staff. Ostensen (39) observed the nature of this support is role dependent, with executives facilitating communication and managers often acting as a bridge between executives and employees. A targeted and nuanced approach to mobilizing the support of organizational leaders is therefore an important aspect of facilitating the recommended improvements in best care for people with obesity identified by participants. 
The focus on care transitions for patients with obesity identified in this study is a novel finding, which has received relatively little attention in previous research. Discharge from hospital is the only care transition studied to date, while participants in this study also discussed admission and intra-service care transitions. A study of transitions from hospital to nursing homes for morbidly obese patients (45) found one third of these discharges were unsuccessful due to funding, staffing and equipment related barriers. However, these challenges may be amenable to intervention, with an evaluation of a package of home care and diabetes education for older adults with obesity (46) found to decrease rehospitalisation rates up to 90 days.

All participants offered recommendations to improve best care for people with obesity, which suggests there is still significant tension for change within this organisation (47). In regards to change management, Kotter stated 'visible crises can be enormously belpful in catching people's attention and pushing up urgency levels' (48), and participants confirmed the care of people with obesity is perceived to be a pressing and prevalent issue. Their recommendations ranged from the very broad to quite specific, and their implementation would require a multidimensional approach. Schein outlined a model of organisational culture inclusive of three layers (49), visible representations of organisational culture (including policies, procedures and physical environment); values underpinning the organisational culture; and assumptions and beliefs held by the workforce. The recommendations to arise from this study include actions that address all of these layers, suggesting that leadership and management clinicians understand the complexity of the task ahead of them.

\section{Limitations}

The most significant limitation for this study was its conduct within a single healthcare organisation. While this was appropriate to explore best care for people with obesity within this specific context, this must be taken into account when considering the potential for generalising these findings.

\section{Conclusions}

The experience and perceptions of these clinical leaders and managers confirm that providing care for people with obesity has significant policy and practice implications. The four themes identified in this study-resource allocation, service context, care transitions and recommendations for best care for people with obesity-described the organisational issues they prioritised in their respective roles. These issues cannot be considered in isolation, as significant overlap and interdependence was evident. Despite the challenges and complexity they perceived, the participants also described the positive outcomes and progress which could be achieved when organisations take direct action to improve the care they provide to people with obesity.

\section{Acknowledgments}

We thank Shelley Karageorge-RN participated in editing of the manuscript.

Funding: None.

\section{Footnote}

Reporting Checklist: The authors have completed the MDAR and COREQ reporting checklist. Available at http://dx.doi. org/10.21037/jhmhp-20-98

Data Sharing Statement: Available at http://dx.doi. org/10.21037/jhmhp-20-98

Conflicts of Interest: All authors have completed the ICMJE uniform disclosure form (available at http://dx.doi. org/10.21037/jhmhp-20-98). The authors have no conflicts of interest to declare.

Ethical Statement: The authors are accountable for all aspects of the work in ensuring that questions related to the accuracy or integrity of any part of the work are appropriately investigated and resolved. The study was conducted in accordance with the Declaration of Helsinki (as revised in 2013) and the Harmonized Tripartite Guideline for Good Clinical Practice from the International Conference on Harmonization. The study was approved by the Western Health Low Risk Ethics Panel (HREC/18/ $\mathrm{WH} / 47094)$ and informed consent was taken from all the patients.

Open Access Statement: This is an Open Access article distributed in accordance with the Creative Commons Attribution-NonCommercial-NoDerivs 4.0 International License (CC BY-NC-ND 4.0), which permits the noncommercial replication and distribution of the article with 
the strict proviso that no changes or edits are made and the original work is properly cited (including links to both the formal publication through the relevant DOI and the license). See: https://creativecommons.org/licenses/by-nc-nd/4.0/.

\section{References}

1. World Health Organisation. Obesity. 2019. Available online: https://http://www.who.int/topics/obesity/en/

2. National Health Service. Statistics on Obesity, Physical Activity and Diet, England, 2019. London: NHS, 2019.

3. The Global Burden of Metabolic Risk Factors for Chronic Diseases Collaboration. Cardiovascular disease, chronic kidney disease, and diabetes mortality burden of cardiometabolic risk factors from 1980 to 2010: a comparative risk assessment. Lancet Diabetes Endocrinol 2014;2:634-47.

4. Australian Institute of Health and Welfare. Data sources for monitoring overweight and obesity in Australia. Cat. no. PHE 244. Canberra: AIHW, 2019.

5. World Health Organisation. Body Mass Index - BMI. Geneva: World Health Organisation, 2020. Available online: https://www.euro.who.int/en/health-topics/ disease-prevention/nutrition/a-healthy-lifestyle/bodymass-index-bmi

6. Rubino F. From bariatric to metabolic surgery: definition of a new discipline and implications for clinical practice. Curr Atheroscler Rep 2013;15:369-76.

7. Crocker AF, Smith SN. Person-first language: are we practicing what we preach? J Multidiscip Healthc 2019;12:125-9.

8. Ross R, Berentzen T, Seidell J, et al. Does the relationship between waist circumference, morbidity and mortality depend on measurement protocol for waist circumference? Obes Rev 2008;9:312-25.

9. Nowicki T, Burns C, Fullbrook P, et al. Changing the mindset: an inter-disciplinary approach to management of the bariatric patient. Collegian 2009;16:171-5.

10. Bucher Della Torre S, Courvoisier D, Saldarriaga A, et al. Knowledge, attitudes, representations and declared practices of nurses and physicians about obesity in a university hospital: training is essential. Clin Obes 2018;8:122-30.

11. Hossain MA, Amin A, Paul A, et al. Recognizing obesity in adult hospitalized patients: a retrospective cohort study assessing rates of documentation and prevalence of obesity. J Clin Med 2018;7:203.

12. Australasian Health Infrastructure Alliance. Australasian
Health Facility Guidelines. Australasian Health Infrastructure Alliance. 2015. Available online: https:// aushfg-prod-com-au.s3.amazonaws.com/download/ Part\%20C\%2003\%20Space\%20Standards_5.pdf (Accessed August 31, 2018).

13. Wignall D. Design as a critical tool in bariatric patient care. J Diabetes Sci Technol 2008;2:263-7.

14. Hales C, Coombs M, de Vries K. The challenges in caring for morbidly obese patients in intensive care: a focused ethnographic study. Aust Crit Care 2018;31:37-41.

15. Cowdell F, Radley K. What do we know about skinhygiene care for patients with bariatric needs? Implications for nursing practice. J Adv Nurs 2014;70:543-52.

16. Mathison CJ. Skin and wound care challenges in the hospitalized morbidly obese patient. J Wound Ostomy Continence Nurs 2003;30:78-83.

17. Blackett A, Gallagher S, Dugan S, et al. Caring for persons with bariatric health care issues: a primer for the WOC nurse. J Wound Ostomy Continence Nurs 2011;38:133-8; quiz 9-40.

18. Gallagher S, Hilton T, Monaghan H, et al. Safe patient handling \& movement: bariatric considerations. Am J Safe Patient Handl Mov 2014;Suppl:S1-16.

19. Beitz JM. Providing quality skin and wound care for the bariatric patient: an overview of clinical challenges. Ostomy Wound Manage 2014;60:12-21.

20. Hyun S, Li X, Vermillion B, et al. Body mass index and pressure ulcers: Improved predictability of pressure ulcers in intensive care patients. Am J Crit Care 2014;23:494-500.

21. New South Whales Health. Work health and safety - management of patients with bariatric needs. North Sydney: New South Whales Health. 2018. Available online: https://www1.health.nsw.gov.au/pds/ ActivePDSDocuments/GL2018_012.pdf (Accessed Aug 31, 2018).

22. Muir M, Heese GA. Safe patient handling of the bariatric patient: sharing of experiences and practical tips when using bariatric algorithms. Bar Nurs Surg Pat Care 2008;3:147-58.

23. Hignett $S$, Griffiths P. Risk factors for moving and handling bariatric patients. Nurs Stand 2009;24:40-8.

24. Berrios LA. The ABCDs of managing morbidly obese patients in intensive care units. Crit Care Nurse 2016;36:17-26.

25. Green SM, Gillett A. Caring for patients with morbid obesity in hospital. Br J Nurs 1998;7:785-92.

26. Leen MP. Establishing a comprehensive bariatric protocol. 
Nurs Manage 2010;41:47-50.

27. Cowley S, Leggett S. The bariatric journey in Australia: hospital case study. Australian Government Australian Safety and Compensation Council. 2009. Available online: https://docplayer.net/21814439-The-bariatric-journeyin-australia-hospital-case-study.html (Accessed August 24, 2018).

28. Thomas SA, Rickabaugh B. Bariatric nurse coordinator: carving out a new role in bariatrics. Bar Nurs Surg Pat Care 2008;3:63-72.

29. Temple G, Gallagher S, Doms J, et al. Bariatric readiness: economic and clinical implications. Bariatric Times 2017;14:10-6.

30. Health W. Western Health Strategic Plan 20152020. Footscray: Western Health, 2015. Available online: http://www.westernhealth.org.au/AboutUs/ CorporatePublications/Documents/StrategicPlan/ WesternHealth_StrategicPlan2015.pdf

31. Better Health Plan for the West Partnership. Better Health Plan for the West. Melbourne: Better Health Plan for the West Partnership, 2012.

32. Brown I, Gould J. Qualitative studies of obesity: a review of methodology Health 2013;5:69-80.

33. Gujral H, Tea C, Sheridan M. Evaluation of nurse's attitudes toward adult patients of size. Surg Obes Relat Dis 2011;7:536-40.

34. Lacroix E, Alberga A, Russell-Mathew S, et al. Weight bias: a systematic review of characteristics and psychometric properties of self-report questionnaires. Obes Facts 2017;10:223-37.

35. Malterud K, Siersma VD, Guassora AD. Sample size in qualitative interview studies: Guided by information power. Qual Health Res 2016;26:1753-60.

36. SocioCultural Research Consultants LLC. Dedoose Version 8.0.35, web application for managing, analyzing, and presenting qualitative and mixed method research data. Los Angeles: SocioCultural Research Consultants LLC, 2018.

37. Tong A, Sainsbury P, Craig J. Consolidated criteria for reporting qualitative research (COREQ): a 32-item checklist for interviews and focus groups. Int J Qual

doi: 10.21037/jhmhp-20-98

Cite this article as: Qvist A, Pazsa F, Hitch D. Perceptions of clinical leaders and managers of inpatients with obesity in an Australian public health service. J Hosp Manag Health Policy 2021;5:15.
Health Care 2007;19:349-57.

38. Henderson R, Rheault W. Appraising and incorporating qualitative research in evidence-based practice. J Phys Ther Educ 2004;18:35-40.

39. Ostensen M. On how and why line managers are drivers of implementation: a qualitative study exploring the behaviours of line managers in the implementation of an organisational health intervention. Trondheim: Norwegian University of Science and Technology, 2018.

40. Akinnusi ME, Pineda LA, El Solh AA. Effect of obesity on intensive care morbidity and mortality: a meta-analysis. Crit Care Med 2008;36:151-8.

41. Lumley E, Homer C, Palfreyman S, et al. A qualitative study to explore the attitude of clinical staff to the challenges of caring for obese patients. J Clin Nurs 2015;24:3594-604.

42. Schneider PL, Li Z. Ethical challenges in the care of the inpatient with morbid obesity. Narrat Inq Bioeth 2016;6:143-52.

43. Stevens J. Definition and classification of obesity. In: Brownell K, Walsh B, editors. Eating disorders and obesity. London: Guilford Publications, 2017:441-6.

44. Hitch D, Pepin G, Lhuede K, et al. Development of the translating allied health knowledge (TAHK) framework. Int J Health Policy Manag 2019;8:412-23.

45. Bradway C, Felix HC, Whitfield T, et al. Barriers in transitioning patients with severe obesity from hospitals to nursing homes. West J Nurs Res 2017;39:1151-68.

46. Whitehouse CR, Sharts-Hopko NC, Smeltzer SC, et al. Supporting transitions in care for older adults with type 2 diabetes mellitus and obesity. Res Gerontol Nurs 2018;11:71-81.

47. Damschroder LJ, Aron DC, Keith RE, et al. Fostering implementation of health services research findings into practice: a consolidated framework for advancing implementation science. Implement Sci 2009;4:50.

48. Kotter J. Leading Change. Boston: Harvard Business School Press, 1996.

49. Schein E. Organisational culture and leadership. 2nd ed. San Francisco: Jossey-Bass, 1992. 